Accelerator Department:

BROOKHAVEN NATIONAL IABORATORY

Associated Universities, Inc.

Upton, New. York

AGS :DIVISION TECHNICAL NOTE

№: 118

E.C. Raka

March 3, 1976

THE TRANSVERSE: COHERENCE DAMPERS

\title{
Introduction:
}

Since March 1973 a feedback damping system has been used to: control the vertical transverse instability present, below the transition energy. of $\approx 8 \mathrm{GeV}$; during normal operation of the Brookhaven AGS at intensities greater than $\approx 4.5 \times 10^{12} /$ pulse. Approximately one year later provision was a lso made to damp an incipient instability. in the horizonta 1 plane which could arise at somewhat higher intensities. Both systems are essential to the operation of the AGS at high intensities i.e: $>6 \times 10^{12} /$ pulse.

The present damping systems are based on a prototype which was used in early studies of the transverse instability. ${ }^{1}$. Details of the design: requirements are given in Ref. 1. To summarize the basic principa 1 , one senses the motion of the center of charge of the beam by means of pick-up electrodes. . This signa 1 is filtered, amplified, and transmitted to a pair of deflecting coils located $\approx 1 / 4$ of a betatron wavelength downstream. The system is narrow band in that it can damp only the lower order coupled bunch modes, $\mathrm{n}=9,8,10 \mathrm{i} . \mathrm{e}$. those modes where the bunched beam acts as a dc beam.

For these modes the spectrum of the difference signal will contain the frequencies $(n-v) f_{0}, M_{0} \pm(n-v) f_{0}, 2 M f_{0} \pm .(n-v) f_{0}$ etc.; where $M$ is the number of bunches and $f_{0}$ the rotation frequency. If we assume that the resistive wa 1 impedance is responsible for the instability the lowest frequency line will predominate: " Clearly, however, significant antidamping at the other frequencies should be avoided. If the bunches are unequal in intensity, then some signal at $\mathrm{mf}_{0} \pm(n-v) f_{0}$, where $m=1$ to $(M-1), \ldots(M+1)$ to $(2 \mathrm{M}-1)$, etc., wil1 also be present. These lines wil1:also appear if 
any of the bunches oscillate independently... However, the relative amplitudes would be larger in this case. Damping of these oscillations will in general not occur since the narrow band system sees only the average posi-... tion of all the bunches and hence the phase of the resultant signal will not be correct:

\section{Description. of the System}

A block diagram of the feedback damping system is shown in Fig. 1. The cathode followers are mounted on the vacuum chamber and consist of a 6922 (E88CC) connected in paralle1 whose input capacity of $\approx 11 \mu \mu \mathrm{f}$ in. series with the $40 \mathrm{~K}$ resistors forms the single filter in the 10op. This attenuates the high frequency components of the bunched:signal which would otherwise over-drive the tubes at high beam intensities. A 3 db point of $\approx 380 \mathrm{kc}$ provides adequate margin for satisfactory damping of the $\mathrm{n}=9$ mode for all reasonable values of tune below the transition :energy.

The difference amplifier is a HA733IC operated at a single ended gain of $\approx 28$. An Analogue Devices $46 \mathrm{~J}$ or $\mathrm{K}$ is used as a summing amplifier line driver so that the overa 11 gain is $\approx 55$ to $>12 \mathrm{MHz}$. The coil driver consists of two. 8233 tubes in para1le1 giving a current gain of $\approx 82 \mathrm{~mA} / \mathrm{V}$ to we11 beyond the self-resonant frequency of the coils $(\dot{\approx}: 4.4 \mathrm{MHz})$.

In the spring of 1974 a new coil assembly was installed providing a two-turn horizontal as well as a two-turn vertical coil. The leads were brought out at the upstream end rather than downstream and the coils have the following dimensions. Both are 92 in. 1ong with the vertical wires separated by 4 in and located at \pm 1.25 in. about the chamber center while the horizonta 1 wires are separated by 4.25 in. and are located at \pm 2 in. above and below the chamber center. On the median plane then the vertical coils $:$ has a strength of $\approx .11$ gauss/amp and hence it can produce a transverse $\Delta \mathrm{p}=\mathrm{B} Z=.77 \times 10^{-5} \mathrm{BeV} / \mathrm{c}$ amp. The horizontal coil has a field that varies somewhat more with the radial position of the beam. We take the average strengths over a 2.5 in aperture as 0767 gauss/amp giving a $\Delta \mathrm{p}=$ $.54 \times 10^{-5} \mathrm{BeV} / \mathrm{c}$ amp.

A differential gain adjustment is provided by employing a FET in . . series with a IK resistor to form a variable attenuator at each input to the 733. An operational amplifier provides the necessary inversion so that a single input can vary the gain in opposite directions in each channel. This is used to balance out the signal due to anyorbit error 
at the PUE location... For the horizontal, of course, a null can on $1 \mathrm{y}$ be obtained for one position of the beam. In principal the gain could be programmed as a function of beam positive but this has not been necessary so far.

Initially the pick-up electrodes were located at straight section $B-3$ and the deflecting coils at $B-10 \ldots$ Since injection is at : A-20 the radiation was rather high in the vicinity of the difference amplifier which was located on the AGS floor next to the main magnet girider. In January 1975 the entire system was moved to $\mathrm{A}-3$ and $\mathrm{A}-10$ where the radiation is minimal. Since that time no component. failure has occurred as opposed to at least one definite malfunctioning of a 733 in the old location.

\section{System Performance}

The damping rate of the feedback loop can be written as ${ }^{1}$

$$
D=\frac{\Delta p}{p} \frac{v \sin \left[n \theta-(n-v) \omega_{0} \cdot T\right]}{4 \pi v 4 \pi \bar{y}_{0}(y-T)}
$$

Where $\Delta \mathrm{p}$ is the transverse momentum imparted by the coils, $\mathrm{p}$ the beam. momentum, $v$ its velocity, $v$ the betatron wave number, $\omega_{0}$ the beam angular frequency, $\theta$ the angular separation between the pick-up electrodes and the deflecting coils; $T$ the signa 1 time delay between these two points and $\bar{y}_{0}(t-T)$ the average position of the beam as seen by the electrodes. One: can also define $\alpha=2 \pi \mathrm{D} / \omega_{0}$ such that any initial displacement of the beam will decay like $\mathrm{e}^{-\alpha \mathrm{N}}$ where $\mathrm{N}$ is the number of revolutions after the perturbation $(|\alpha| \ll<\mid 1)$. TThen wecan writeiinvilewof thesabove

$$
\alpha=\frac{\Delta p}{p} \cdot \frac{\bar{\beta}}{2} \cdot \frac{\sin \left[n \theta-(n-v) \omega_{0} T\right]}{\bar{y}_{0}(t-T)}
$$

where $\bar{\beta}=R / v$.

In the above equations we have written $\bar{y}_{0}$ to imply that the beam posi-.. tion is measured at $\beta=\bar{\beta}$ in an $A G$ machine: If this is not true as in the present case we can write $\bar{y}_{0}=\sqrt{\bar{\beta} / \beta_{0}}$ y. where $\beta_{0}$ is the" value of $\beta$ at the PUE location... Since the coil is located about the center of a $10 \mathrm{ft}$ straight section where $\beta_{1}=\bar{\beta}$ we can write (1a) as

$$
\alpha=\frac{\Delta p}{p} \frac{\sqrt{\beta \cdots \beta_{o}}}{2} \frac{\sin \left[n \theta-(n-v) \omega_{o} T\right]}{y_{o}(t-T)} .
$$


Now $\Delta \mathrm{p}=\mathrm{Ky}_{\mathrm{o}}$, where $\mathrm{K}$ depends upon the pick-up electrode sensitivity, the: beam intensity, and the gain characteristics of the electronics including the deflection coils. The damping rate $D$ is then given by

$$
D=\sqrt{\beta_{0}} \cdot \frac{K}{4 \pi p_{v}} v \sin \varphi=\frac{K \omega_{\infty} \sqrt{\beta_{0}} \sin \varphi}{4 \pi \gamma m_{0} c}
$$

where $\varphi=\left[\mathrm{n} \theta-(\mathrm{n}-\vartheta) \omega_{0} \mathrm{~T}\right]$ and $\omega_{\infty}=: \mathrm{c} / \mathrm{R}$. Also we can write

$$
\alpha=\frac{\sqrt{\beta_{0}}}{2 \mathrm{p}} \mathrm{K} \sin \varphi
$$

for the damping rate per revolution. The maximum damping rate $(\sin \varphi \approx 1)$ thus depends upon.K for a given beam momentum. $\mathrm{p}$. : If we ignore for the moment the frequency characteristics of the filter then $\mathrm{K}$ is just the gain from pick-up electrodes to deflection coils in units of momentum/displacement. We have for the vertica 1 PUE" differential sensitivity $\approx .08$ volts/cm per $10^{12}$ protons and for the horizontal $\approx .096$ volts $/ \mathrm{cm} 10^{12}$. Thus

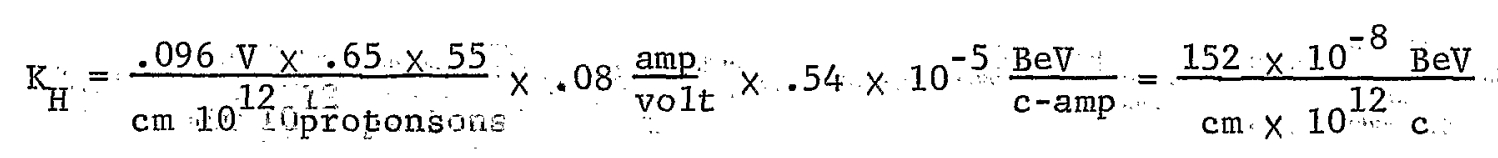

where .65 is the gain of the cathode followes into $180 \Omega$ and

$$
\mathrm{K}_{\mathrm{V}}=.08 \frac{\mathrm{volt}}{\mathrm{cm} \times 10^{12}} \times .65 \times 55 \times .082 \frac{\mathrm{amp}}{\mathrm{volt}} \times .77 \times 10^{-5} \frac{\mathrm{BeV}}{\mathrm{amp}}=\frac{181 \times 10^{-8} \mathrm{BeV}}{\mathrm{cm} 10^{12} \mathrm{c}}
$$

The maximum damping rate at $\gamma=1.7$ and $5 \times 10^{12}$ protons is ..

$$
D_{\nabla}=\frac{181 \times 10^{-7}}{2 \times 1.7 \times .938 \mathrm{BeV} / \mathrm{c}} \frac{\mathrm{BeV}}{\mathrm{c} \mathrm{cm}} \cdot \frac{.371 \times 10^{6} \sqrt{14.721 .8} \times 10^{2} \mathrm{~cm}}{\mathrm{sec}}=3769 \mathrm{sec}^{-1}
$$

and.

$$
D_{\mathrm{H}}=\frac{152 \times 10^{-7}}{2 \times 1.7 \times .938} \times .371 \times 10^{8} \sqrt{14.710 .5}=2197 \mathrm{sec}^{-1}
$$

The maximum observed vertical growth rate in the absence of damping was $\approx 500 \sec ^{-1}$ at a $\gamma \approx 1.65$ and an intensity $>6 \times 10^{12}$. Hence we have a damping rate about eight times the observed growth rate since both are 
directly proportional to the beam intensity. In the horizontal plane the growth rate when the instability occurs has always been less than twice the vertical value at the same intensity.... Thus the horizontal system gain is also quite adequate. These growth rates are for the $(9-v)$ coupled bunch mode in both cases. It should be noted however that "m" the within the bunch mode number is zero for the vertical oscillations and one for the horizontal due to the much larger value:of $\xi=(\mathrm{d} v / \mathrm{v}) /(\mathrm{dp} / \mathrm{p})$, the chromaticity, in the horizontal plane than in the vertical plane.

Now let us fird the maximum $\alpha$ at injection $(\gamma=1.213)$ for $10^{13}$ and the $(9-\vartheta)$ component of any initial displacement from the equilibrium orbit. We obtain

$$
\alpha=\frac{\sqrt{14.7 \times 2.1 .8} \times 10^{2} \times 181 \times 10^{-7}}{2 \times 1.213 \times .938}=.0142 \ldots
$$

Thus it would take about 70 revolutions or $\approx 340 \mu \mathrm{sec}$ for the $(9-v)$ component to be reduced to $1 / \mathrm{e}$ of its initial value. This is comparable to. the spiraling time of the unaccelerated beam with normal $\dot{B}$. With multi-.. turn injection however successive revolutions will have different phases of. oscillation so there is no way that the system can adequately control injection errors... In general the system has negligible effect at injection and also at high energy (due to the $1 / \gamma$ term in $D$ or $\alpha$ ) so it is allowed. to remain sensitive at all times.

Next let us consider how $\varphi$ varies in the AGS. For the A-3, A-10.". spacing. we have $\theta=10.7^{\circ}$ and $\mathrm{T} \cong 125 \mathrm{nsec}$ ( 100 for the cable and $25_{\text {: for }}$ the electronics) for the feedback 1oop. For $\gamma=1.7 \mathrm{f}_{0} \cong 300 \mathrm{kc}$ and if $w=8.8$ we obtain for $n=9$ a $\varphi=93.6^{\circ}$ and $60 \mathrm{kc}$ as the principal frequency in the difference signa 1 spectrum. At $\psi=8.6, \varphi=90.9$. with $120 \mathrm{kc}$ as the principal frequency and for $y=8.9, \varphi \cong 95^{\circ}$ and $\mathrm{the} \mathrm{n}=9$ mode frequency is $30 \mathrm{kc}$. The lowest observed coherent frequency was $\approx$ $13 \mathrm{kc}$ for a $\gamma \cong 1.3$ and a $v_{\mathrm{x}} \approx 8.94$ for which $\varphi \cong 96^{\circ}$. Thus we see that for the $n=9$ mode there is no significant variation in $\varphi$ over: the norma 1 range: of AGS parameters.

In order to include the effect of the filter on the damping rate we must multiply $K$ by $\left(e^{\left.-i \text { arc } \tan f / f_{c}\right) /{\sqrt{1+\left(f / f_{c}\right)}}^{2}}\right.$ where $f_{c} \cong 380 \mathrm{kc}$ and $f=|n-v| f_{0}$... Thus at $60 \mathrm{kc}$ the gain is still maximum but the total phase is $\Phi=\varphi+\arctan \left(f / f_{c}\right)=103.6^{\circ}$ and $\sin \Phi=.972$ so that the damping 
rate is essentially the same as quoted above. For $120 \mathrm{kc} \Phi=108.4^{\circ}$ and sin $\Phi \cong .95$ while the gain $\mathrm{K}$ is reduced by about $5 \%$ and the overall damping rate by $\approx 10 \%$. At these frequencies the damping coils and driver contribute no significant phase lag.

With the system as described above adequate damping was obtained at intensities up to $\approx 7 \times 10^{12}$ protons/pulse. During most of 1974 the AGS was operated with the horizontal tune above the vertical tune from injection until $\gamma \cong 1.5$. At times $v_{\mathrm{x}}>8.9$ and at intensities near $7 \times 10^{12}$ the horizontal damper could not adequately control the resulting instability. In early 1975 the horizontal tune was Iowered to $\leqq 8.7$ and the vertical was kept in the region of 8.8-8.9. Under these conditions the horizontal instability was essentially absent at $7 \times 10^{12}$ but above this value occasional blow-up in the vertical plane could occur. This was suppressed by programming the vertical sextupoles at about $6 \mathrm{~A}$ starting 10 to $15 \mathrm{msec}$ after injection. With this combination intensities of $9.8 \times 10^{12}$ were reached. It should be noted that the above sextupole current doubled the vertical chromaticity at $\gamma \approx 1.5$ and decreased the honizontal value $\approx 25$ $30 \%$. The horizontal instability returns at intensities $\gtrsim 7 \times 10^{12}$ under these conditions but the damper was abieto suppress it up to the $9.810^{12}$ intensity peak achieved prior to February 19, 1976.

Observations made on the nature of the vertical blow-up during the rare pulses when it occurred indicated that a significant signal at the $\left(9-v_{y}\right)$ frequency was present which grew. with the instability. This could also be seen on the envelope of an rf difference signal showing the individual bunches. It: should be remarked that with the damper turned off; coherence would occur on every pulse with a faster growth rate and starting closer to the injection energy. This behavior along. with the higher intensity: needed for the growth to occur"suggested that it might be due to the damping system itself. See Appendix C for discussion of this point. In order to test this possibility the response of the loop was modified so as to give a $12 \mathrm{db}$ per octave roll-off for frequencies $\approx 25 \mathrm{kc}$ or greater. The sense of the feedback was reversed ${ }^{3}$. because now $\Phi \cong \pi+\varphi$ and the overa 11 gain was changed so that $|\mathrm{k}|$ was the same as before at $\approx 42.7 \mathrm{kc}$. Details of the change are described in Appendix A.

In late January 1976 this modification was tested in the vertical loop.. It was immediately apparent that the performance had been improved. 
since it was possible to operate at intensities $>8 \times 10^{12}$ without the sextupole program and without any vertical blow up.. Subsequently, an intensity of $1.03 \times 10^{13}$ was reached in early February 1976 again without the need of sextupoles.

\section{Conclusion}

By putting in the $12 \mathrm{db} /$ octave filter the gain for higher order modes is reduced so drastically that any damping or antidamping action by the system is negligible. Thus as the AGS intensity increases one still might expect growth in one of the higher order coupled bunch modes or in case of unequal bunch population, individual bunch instabilities. 2,7

If such instabilities do eventually occur at intensities $>10^{13}$ then some nominal, control is possible using the sextupoles to vary the chromaticities, but nothing further can be expected" from the present damping system. An :entirely new system with wide band electronics operating on : the individual bunches would have to be employed:

In closing I should acknowledge the invaluable assistance of Mr. E. Gi11 in carrying out this work.

\section{References:}

1. E. Raka;" "Damping Coherent Oscillations in the AGS", IEEE Trans. Nuc1. Sci. NS-14, No ...3, 1091, (1967).

2.: F. Sacherer, "Transverse Bunched Beam Instabilities", Proc. 9th, Int: Accel. Conf., p.347 (1974):

3. G.H. Reese, "Servo Theory for the Control of Coherent Betatron Oscillations in Nimrod"!, NIRL/R/99:(1965).

4. E. Courant, H. Snyder, "Theory of the Alternating Gradient Synchrotron", Annuals of Physics, Vo1.. 3, No .: 1 (1958)...

3

$\mathrm{ECR} / 1$ sk

Distr: Dept. Admin. MCR Operators Oper. Coord. Accel. Dept. Physicists. 
Appendix A

The loop response was modified as follows... For the input RC to the cathode followers $R$ was changed to $401: K \Omega$ and $C$ to $68 \mu \mu$ plus the $\approx 11 \mu f$ shunt capacity. Thus $\omega \mathrm{C}_{i} \mathrm{R}_{i}=1$ for $5 \mathrm{Kc}$ and $\mathrm{it}$ was decided to control the low frequency response by making the output coupling capacitor driving the terminated $180 \Omega$ cable $.176 . \mu$. This also results in $\omega \mathrm{C}_{c} R_{c}=1$ at $5 \mathrm{Kc}$ (a11 other low frequency time constants being $\gg 31.7 \mu \mathrm{sec}$ ). The response then can be written as

$$
\frac{A e^{j \theta}(f / f / f)}{1+\left(f / f(f)^{2}\right.}
$$

where $f_{c}=5 \mathrm{Kc}$ and $\theta=\pi / 2-\cdots 2$ arctan $(\mathrm{f} / \mathrm{f})$ where negative $\theta$ represents a phase $\mathrm{lag}$ and $\mathrm{A} / 2$ is the gain at $f_{\mathrm{C}}(\approx .36)$. Next the $\mu \mathrm{A} 733$ gain was increased to the maximum possible $i_{. e .} \approx 180$ single ended. Then the $46 \mathrm{~J}$. was used as a combination differentiatón, integrator, summing amplifier. If we cal1 $R_{D} C_{D}$ the differentiation elements and $R_{i} C_{i}$ the integration elements then they were chosen so that $R_{D} C_{i}=R_{i} D_{d}=T_{c}=31.7 \mu \mathrm{sec}$ with $R_{D} / R_{i}=$ 10 and $R_{D}=39 \mathrm{~K}$. With $\mathrm{O}_{\mathrm{D}}=8200 \mu \mu \mathrm{f}$ and $\mathrm{C}_{i}=820 \mu \mu \mathrm{f} \mathrm{f}_{\mathrm{c}}=1 / 2 \pi \mathrm{T} \cong \mathrm{Kc}:$ we have for the gain of this stage

$$
\frac{2 \times 10 e^{j \theta}(f / f / f c)}{1+(f / f)^{2}}
$$

where $\theta$ is the same as above and the factor of two comes from summing both outputs of the 733 . Thus the total gain is $\approx$

$$
\frac{25900 e^{j 2 \theta}(f / f / f c)^{2}}{\left[1+(f / f)^{2}\right]^{2}}
$$

which at $42 \mathrm{KC}$ is $\approx 35.7$ i.e. the nominal Iow frequency gain of the previous configuration. At this frequency $2 \theta=152.8^{\circ}$ of 1 ag so for the $n=9$ mode $\varphi=94.4^{\circ}$ and $\Phi=-180^{\circ}+152.8+94.4=67.2^{\circ} \cdots$ where we subtracted $180^{\circ}$ because the sense of the feedback was reversed. Thus the damping rate for $42 \mathrm{Kc}$ is $\approx 92 \%$ of the maximum values calculated earlier: It is obvious 
how the gain scales with frequency but let us compare the value at $3.6 \mathrm{MHz}$ with the earlier configuration. We obtain $\approx .005$ for this combination vs 3.75 for the previous filter. Due to stray capacities this large a reduction is not achieved but it is still greater than two orders of magnitude.

As a fina liexample, let us calculate the damping rate at $\mathrm{f}=13 \mathrm{Kc}$. for the $n=9$ mode. The gain is 290 and $2 \theta=95.85^{\circ}$ so that $\phi=12.15^{\circ}$ and $\sin$ : $\Phi=.21$ so the damping rate is $.21 \times 290 / 35.7 \cong 1.71$ larger than the maximum calculated earlier. Because of the higher gain of this system in the region around $5 \mathrm{Kc}$, care must be taken to minimize noise pick-up or generation (by the 6922 tubes) at these frequencies.

Fina 11ywe should remark that because the input RC to the cathode followers was increased by $a$ factor of $\approx 70$ the high frequency components. of the input signal are greatly attenuated so there should be no chance of overdriving them at intensities much larger than $10^{13} \cdots$ This reduction is so great that even though the 733 gain was increased somewhat there seems to be. no further need for the variable attenuators at its input since any: difference signal due to an orbit error wil1 be negligible. At present the above modification has been installed in both the vertica 1 and horizonta 1 damping: loops. 


\section{Appendix $\cdot B$}

\section{Orbit Bump Due to Damping Coils:}

There is always a dc current flowing in the two-turn deflecting coils of $\approx 130 \mathrm{~mA}$. The resulting orbit deflection is given by ${ }^{4}$

$$
y(\varphi)=\frac{\beta^{\frac{7}{2}} \cdot \eta}{2 \cdot \sin \pi \eta} \cdot \int_{\varphi}^{\varphi+2 \pi} f(\Psi) \cdot \cos \cdot(\pi+\varphi-\Psi) \cdot d \Psi
$$

where $\varphi=\int \mathrm{ds} / \beta_{\eta}$. For our two-turn coil of length: $\ell$ centered about $\Psi=0$. we can write.

$$
y(\varphi)=\frac{\beta^{\frac{1}{2}}(\varphi)}{2 \sin : \pi \nu} \frac{\Delta B}{B_{\rho}} \int_{-\ell / 2}^{\ell / 2} \cos /(\pi+\varphi) \beta^{\frac{1}{2}} d s
$$

where $B_{\rho}$.is the momentum of the protons and $\Delta B$ the field due to the coil At $200 \mathrm{MeV} \mathrm{B}_{\rho}=2.15 \times 10^{3} \mathrm{Kg} \mathrm{cm}$ and for the vertical deflecting coil $l=$ $233 \mathrm{~cm} . \triangle B=.11$ gauss/amp and for $\beta$ we take the average value at the center. of the $\operatorname{coi} 1 \bar{\beta}=R /$ g so that

$$
y(\varphi)=\frac{\sqrt{\beta(\varphi) \bar{\beta}}}{2 \sin \pi v} \cos v(\pi+\varphi) 1.55 \times 10^{-6}
$$

If $v \varphi \approx \pi / 2$ then we can be clise to a $\beta_{\max } \cong 22$ meters vertically and we obtain

$$
\mathrm{y}_{\max } \cong-\frac{1}{2} \sqrt{22 \times 15} \times 1.55 \times 10^{-4} \mathrm{~cm}=-1.4 \times 10^{-3} \mathrm{~cm}
$$

and, of course, somewhat less for the horizontal coil. 


\section{Appendix C}

As mentioned earlier, when a bunched beam oscillates in the coupled bunch mode: $\mathrm{n}$ there will be present in the difference signal spectrum the: frequencies $(n-\vartheta) f_{0},[M \pm(n-v)] \cdot f_{0},[2 M \pm(n-v)] f_{0}$, etc $\therefore$ depending upon the bunch width. The relative amplitudes of these lines willalso depend upon "m", t, the withinuthe bunch mode number, and the frequency $f_{f}=f_{0} / \eta$ where $\eta=\gamma_{t r}^{-2}-\gamma^{-2} \cdot$ We should also note that these frequencies are to be considered negative or positive depending upon whether the wave velocity $R[M \pm(n-i)]$ (ur. $/[M, n]$ etc. is less than or greater than the particle velocity ${ }_{0}{ }^{2}$ " For M bunches there are only $M$ distinct coupled bunch modes $n=0,1, \cdots(M-1)$ and $2 \pi n / M$ is the phase difference between bunches.

Now, so far we have considered only the loop response at the lowest frequency line for the $\mathrm{n}=9$ coupled bunch mode. The damping rates ca1culated previously are only valid for a DC beam: or one that is not only tigh $1 \mathrm{y}$ bunched and.for which $\xi \approx 0$. Neither of these conditions prevai: in the AGS particular1y in the' region: of $\gamma=1.5-1.7$ where vertical growth could occur with the damper active. In order to calculate the damping rate for the bunched beam we need the loop response at $(21-9)$ f?, $(3+v) f_{o},(33-v) f_{0}$ and $(15+v) f_{0}$ since these frequencies will be present in the spectrum of the difference signal for the "m=0" within the bunch mode. ${ }^{2}$ Assuming as before that $\gamma=1.7$ and $v=8.8$ we obtain for $f=12.2$ f $=3.66 \mathrm{MHz}$ a $\Phi=224.7+83.9+11-159.3^{-}=160.3^{\circ}$. Since $\sin \Phi=.337$ "and the filter gain is 0.1 at this frequency the nominal damping rate is .039 that of the maximum low frequency value.. For $\mathrm{n}=3, \mathrm{f}=11.8 \mathrm{f}=3.54 \mathrm{MHz}$ and $\Phi=32.1+84+11-162=-34: 9^{\circ}$ and $\sin \Phi:=-.572$ which means antidamping. The filter gain is . 11 here so the rate is -.063 times the low frequency value. We have includedi here $11^{\circ}$ of $\mathrm{lag}$ due to the coil and driver in calculating the. $\Phi$ 's. Thensystem response at $23.8 \& 24.2 \mathrm{f}$ is negligible since in addition to the factor of two reduction from the filter these frequencies are we11 above the self resonant. frequency of the coil $(\approx 4.4 \mathrm{MHz})$ and most of the current will be shunted by the stray capacity. 
In calculating the effective damping rate we proceed in a manner.... similar to that used in Ref. 2 for obtaining the growth rate for a bunched beam due to the resistive wal1 impedance. That is we sum the 1 oop response.. over the bunch spectrum with the value at each line weighted by the square of the amplitude (hence the energy.)

$$
D_{0}=\frac{\sum_{n} D\left(f_{n}\right) h_{o}\left(f_{n}-f_{\xi}\right)}{\sum_{n} h_{o}\left(f_{n}-f_{\xi}\right)}
$$

where $h_{0}(f)=|\widetilde{\mathrm{P}}(f)|^{2}$ and $\widetilde{\mathrm{p}}$ is the Fourier transform of the center of charge motion for the $m=0$ mode. It can be written as

$$
\widetilde{p}_{0}=\frac{2 \cos (\pi(u) / 2)}{\left.[1-(\pi) \omega / \pi)^{2}\right]}
$$

where $\pi ;$ is the bunchilength. The frequencies $f_{n}$ are just the five lines: mentioned above where those for $\mathbf{n}=9,21,33$ are considered negative in evaluating $h$ and those for $n=3,15$ a re considered positive. With $l \approx$

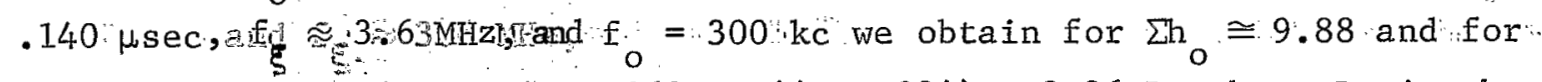
the numerator $D_{\mathrm{V}}(2.5-4 \times .063+\cdots .44 \times .034)=2.26 \mathrm{D}_{\mathrm{v}}$ where $\mathrm{D}_{\mathrm{v}}$ is the maxinum low frequency damping rate calculated previously.. Hence the effective damping rate of the loop for the $n=9$ coupled bunch mode $(m=0)$ for the parameters chosen is $\approx .23: \mathrm{D}_{\mathrm{V}}$ or less than twice the observed growth rate for the gain quoted above... We see that the antidamping for. the $n=3$ line results in on $1 y$ a $10 \%$ reduction of the total. This rate will not be significantly different at lower values of $\gamma$ where a slight ly larger value is expected.

Since the system functioned satisfactorily ibelow $7 \times 10^{12} \mathrm{protons} / \mathrm{pulse}$ and its gain is $\sim$ to intensity as is the resistive wallgrowth rate, vone cannot explain the anomalous growth of the $n=9$ coupled bunch mode in the range of $\gamma=1.5-1.7$ if the threshold for the instability at these energies is the same for the range of $\gamma=1.25-1.5$. However, if. this is not the case, then the slight expected difference in damping rate noted above when the safety margin is less than two might be an explanation for the occasional growth of this mode. In order to settle this question, a series of growth. rate and threshold measurements would have to be made by turning the damper off at different values of $\gamma$. 
Finally; it should be remarked that the increased vertical chromaticity produced by the vertical sextupoles was "such as to reduce the growth rate for the $m=0$ mode. This, of course, increases $f_{\xi}$ and hence reduces further the effective damping rate. The loop response modifications discussed in Appendix A leaves only one term in the sum :.. of the numerator for $\mathrm{D}_{\mathrm{o}}$, but makes its magnitude much more dependent upon the value of $(9-v) f_{0}$. 
